TITTONELL, P.A.; DE GRAZIA, J.; CHIESA, A. Adición de polímeros superabsorbentes en el medio de crecimiento para la producción de plantines de pimiento. Horticultura Brasileira, Brasília, v. 20, n. 4, p. 641-645, dezembro 2002.

\title{
Adición de polímeros superabsorbentes en el medio de crecimiento para la producción de plantines de pimiento
}

\author{
Pablo Adrián Tittonell; Javier De Grazia; Angel Chiesa
}

Cátedra de Horticultura y Floricultura, Facultad de Ciencias Agrarias, Universidad Nacional de Lomas de Zamora. Camino de Cintura km 2, Llavallol (1836), Buenos Aires, Argentina. E-mail: jadegrazia@yahoo.com; degrazia@agrarias.net

\section{RESUMEN}

Los polímeros sintéticos son aditivos que poseen alta capacidad de retención hídrica y mejoran la eficiencia en el uso del agua por parte de las plántulas. La velocidad con que emergen las plántulas, su uniformidad y su tasa de crecimiento inicial son determinantes para la obtención de plantines de calidad. Existen tres grupos principales de polímeros: co-polímeros de almidón, polivinil alcoholes y poliacrilamidas. Con el objetivo de evaluar la aptitud de los polímeros en la producción de plantines de pimiento, un copolímero de propenamida-propeonato se adicionó a sustratos preparados con y sin materiales compostados y a un sustrato comercial. Se caracterizó el comportamiento del mismo a través de la tasa de crecimiento y características cualitativas de los plantines. La adición del polímero al sustrato permitió mejorar la precocidad, uniformidad y tamaño de plantines de pimiento, especialmente en las mezclas carentes de compost. En dichos tratamientos, la tasa de crecimiento aumentó en mayor medida como consecuencia de un mejor desarrollo foliar, ya que la tasa de asimilación no fue significativamente afectada en todos los casos. La relación vástago/raíz no fue favorablemente afectada por adición del polímero y dependió más del tipo de sustrato empleado. Mediante la adición del polímero empleado los parámetros de calidad del plantín mejoran, ya sea por una mayor retención hídrica, por una mayor capacidad de intercambio iónico, o por ambas razones.

Palabras-clave: Capsicum annuum L., aditivos, retención hídrica, sustrato, compost.

\begin{abstract}
Hydrophilic polymers added to growing media in pepper transplants production

Water-storing synthetic polymers (soil additives designed to improve plant establishment and growth in arid environments) has been shown to improve the growth of horticultural plants. Previous research has shown that synthetic polymers are useful when added to low nutrient-holding and water-retaining growing media. There are three groups of polymers used in these applications, some of them added with nutrients and growth starters. A propenamidepropeonate co-polymer was added to substrates containing different levels of compost to evaluate its advantages in pepper transplant production. We evaluated growth patterns and quality traits of pepper seedlings, grown on three peat-based growing media. Seedlings earliness, uniformity and size were improved by polymer addition, especially to low compost substrates. Shoot:root/ratio was not improved by polymer addition but by substrate characteristics. Growth rate was more dependent on leaf area development than on assimilation rate. Seedlings quality traits tended to be improved by polymer addition. One explanation for polymer-treated substrate performance seems to be that nutrients held within the matrix of the polymer are less prone to leaching.
\end{abstract}

Keywords: Capsicum annuum L., additives, water retention, substrate, compost.

(Recebido para publicação em 17 de outubro de 2001 e aceito em 10 de abril de 2002)

\section{Tos polímeros sintéticos,} superabsorbentes por su alta capacidad de retención hídrica, son aditivos que fueron diseñados para mejorar el establecimiento y el crecimiento vegetal en suelos de ambientes áridos (El Sayed et al., 1991; Johnson \& Piper, 1997). Al hidratarse, los gránulos secos de los polímeros se gelifican formando partículas que retienen cantidades de agua equivalentes a cientos de veces su peso seco. Los fragmentos de gel resultantes se comportan como pequeños reservorios individuales que presentan la mayor parte de su humedad almacenada en forma disponible para las plantas. La influencia de estos polímeros sobre la dinámica del agua dentro del suelo y su efecto positivo sobre la disponibilidad hídrica para las plantas en los medios de cultivo ha sido ampliamente documentada (Tayel \& El Hady, 1981; Johnson, 1984; Taylor \& Halfacre, 1986; Orzolek, 1993).

La literatura ofrece, además, crecientes evidencias de que el empleo de estos polímeros no sólo es útil bajo condiciones de extremada aridez, sino que algunos de ellos resultan beneficiosos en la producción de especies hortícolas, cultivadas en condiciones de estrés hídrico moderadas (Pill \& Jacono, 1984; Taylor \& Halfacre, 1986) y se ha demostrado que mejoran la eficiencia en el uso del agua en diferentes sistemas de cultivo (El Hady et al., 1981; Azzam, 1985; Johnson \& Leah, 1990). La etapa inicial en el crecimiento de los plantines constituye el momento más crítico en su producción, razón por la cual la misma debe ser completada en forma rápida (Klassen, 1993). La velocidad con que emergen las plántulas, su uniformidad y su tasa de crecimiento inicial son determinantes para la obtención de plantines de calidad y en períodos de tiempo razonables (Liptay \& Nicholls, 1993).

Existen tres grupos principales de polímeros utilizados en estas aplicaciones: co-polímeros de almidón (poliacrilonitril-almidón); polivinil alcoholes (co-polímeros de ácidos vinil alcohol-arílicos) y poliacrilamidas (copolímeros de acrilamida sódicaacrilato). Se han desarrollado polímeros adicionados con nutrientes y precursores de crecimiento que además de mejorar las relaciones hídricas en el medio de 
crecimiento radicular, aportan dichos elementos, y aumentan la capacidad de intercambio de iones que el medio posee (El Hady et al., 1981).

Los sustratos utilizados en la producción de plantines son muy diversos, variando en su complejidad desde turba de Sphagnum pura, o en mezclas de proporciones volumétricas variables con perlita, arenas, micas, materiales compostados o suelo extraído de capas u horizontes superficiales. Estas mezclas suelen ser preparadas por los propios productores o por firmas comerciales. Normalmente se presentan estériles y con sus atributos fisicoquímicos como $\mathrm{pH}$, conductividad eléctrica, densidad aparente, humedad, relación $\mathrm{C} / \mathrm{N}$, capacidad de intercambio iónico ajustados y especificados.

La incorporación de polímeros sintéticos (particularmente de aquellos adicionados con nutrientes) en la mezcla de los sustratos, cuando éstos son preparados en el propio establecimiento, puede mejorar su comportamiento y aún evitar o disminuir la necesidad de agregar compost o tierra. Con el objetivo de evaluar la aptitud de los polímeros en la producción de plantines de pimiento, se realizó un ensayo en el que los mismos se adicionaron a diferentes sustratos para cultivo de plantines en las dosis recomendadas por los fabricantes, utilizando mezclas preparadas con y sin materiales compostados, y un sustrato comercial conteniendo compost, que difieren en sus propiedades físicas y químicas.

\section{MATERIAL Y MÉTODOS}

Se realizó un ensayo en el invernadero destinado a la producción de plantines de un establecimiento comercial de la zona de Las Colonias, partido de Florencio Varela, ubicado en el cinturón hortícola de Buenos Aires, con las siguientes características estructurales: invernadero tipo capilla de $30 \mathrm{~m}^{2}$ (plantinera), con una altura de cumbrera de $2,9 \mathrm{~m}$, estructura de madera y cobertura de polietileno LDT de 150 $\mathrm{m}$ en techo y laterales, con ventilación lateral, donde se mantuvo una amplitud térmica promedio de $6,44^{\circ} \mathrm{C}$ (máxima media $26,23^{\circ} \mathrm{C}$, mínima media $19,79^{\circ} \mathrm{C}$ ) a través del manejo de la ventilación y la calefacción mediante radiadores eléctricos. El comportamiento de los plantines se caracterizó a través de la tasa inicial de crecimiento y de las características cualitativas de los mismos.

El híbrido de pimiento utilizado fue Saxo F1 (Clause Semences International Inc.). Las semillas fueron pregerminadas en cámara a $25^{\circ} \mathrm{C}$, y sembradas con 1,5-2 $\mathrm{mm}$ de radícula emergida. La siembra se efectuó el 28/ $09 / 99$. Se utilizaron bandejas plásticas de $30 \times 50 \mathrm{~cm}$, con una capacidad de 66 celdas de tipo pirámide invertida con un volumen unitario de $57 \mathrm{~cm}^{3}$, una profundidad de $50 \mathrm{~mm}$ y un lado de boca de $40 \mathrm{~mm}$. Las bandejas sembradas fueron ubicadas en mesadas a $1,10 \mathrm{~m}$ de altura, y se regó por micro-aspersión. La fertilización consistió en el agregado de $50 \mathrm{mg} . \mathrm{L}^{-1}$ de fertilizante soluble (15:15:15) semanalmente junto con el riego, desde la plena expansión de la primer hoja verdadera hasta la semana inmediatamente anterior al muestreo final. El plan fitosanitario comenzó con el lavado de las bandejas con una solución acuosa de hipoclorito de sodio (20 g. $\left.\mathrm{L}^{-1}\right)$, previo a su llenado con sustrato estéril, aplicaciones preventivas semanales de sulfato neutro de oxiquinoleína $\left(0,3\right.$ g. $\left.\mathrm{L}^{-1}\right)$ y quincenales de captan $\left(2,5\right.$ g.L $\left.\mathrm{L}^{-1}\right)$.

Los sustratos utilizados fueron: TPC (45\% v/v de turba de Sphagnum, 30\% de perlita y $25 \%$ de material vegetal compostado), TP (60\% de turba de Sphagnum y $40 \%$ de perlita) y un sustrato comercial compuesto por turba de Sphagnum, compost orgánico (40\% $\mathrm{v} / \mathrm{v}$ ), perlita y vermiculita. A cada tipo de sustrato a emplear se le midió su conductividad eléctrica en extracto $\left(25^{\circ} \mathrm{C} ; 1: 2,5 \mathrm{v} / \mathrm{v}\right)$. Todos los medios de crecimiento fueron evaluados con y sin el agregado del polímero, a razón de 6 g. $\mathrm{L}^{-1}$ de sustrato. El polímero adicionado es una mezcla de copolímeros entrelazados de propenamidapropeonato, adicionado con precursores orgánicos e inorgánicos de vitaminas y hormonas vegetales, fertilizantes minerales sintéticos de liberación lenta, fertilizantes orgánicos sintéticos y arenas de dióxido de silicio.
El muestreo para la evaluación de los parámetros de calidad de los plantines se realizó cuando en la mayoría de los tratamientos se alcanzó el tamaño de transplante, 7-8 hojas verdaderas, correspondiendo a los 57 días desde la siembra (dds), y consistió en cinco plantas completas por repetición. Se determinó el peso fresco total (PFT), área foliar (AF) calculada como la sumatoria de la superficie de todas las hojas, altura del vástago $(\mathrm{H})$ y diámetro basal del tallo (DBTal). Las muestras fueron secadas a $65^{\circ} \mathrm{C}$ durante $72 \mathrm{~h}$ en estufa con circulación forzada de aire y se determinaron los pesos en seco de vástago (PSV) y raíz (PSR). Se calculó el peso seco total (PST), la relación vástago-raíz (Rel V/R) y el contenido porcentual de materia seca (MS\%).

Para la análisis del crecimiento se emplearon los procedimientos $\mathrm{y}$ cálculos propuestos por Hunt (1978; 1982) para el procesamiento de los datos obtenidos. Se calcularon mediante relaciones simples la proporción areal de hojas $(P A H=A F / P S T)$ y el área foliar específica $(A F E=A F / P S H)$. Se integró la superficie fotosintéticamente activa presente en la planta en los diferentes muestreos para estimar la duración del área foliar (DAF). La tasa relativa de crecimiento (TCR) fue calculada tanto en relación a la materia seca, mediante la aplicación de la fórmula $T C R\left(\right.$ di $\left.^{-1}\right)=$ $\left(\ln P_{2}-\ln P_{1}\right) /\left(t_{2}-t_{1}\right)$; siendo $\mathrm{P}_{2}$ y $\mathrm{P}_{1}$ los valores de peso seco respectivos a los tiempos $\mathrm{t}_{2} \mathrm{y}_{1}$ (Venus \& Causton, 1979). La primer medición $\left(\mathrm{t}_{1}\right)$ se realizó al momento de la emergencia, 9 dds, mientras que la segunda $\left(\mathrm{t}_{2}\right)$ a los $57 \mathrm{dds}$. Mediante un procedimiento análogo se calculó la tasa de expansión foliar en términos relativos (TEF). La tasa de asimilación neta (TAN) desde la siembra al transplante se calculó en base a los datos obtenidos, utilizando la relación: $T A N\left(\right.$ g. $^{-2} \cdot$ dia $\left.^{-1}\right)=\left(P S T_{\text {final }} / A F_{\text {final }}{ }^{-}\right.$ $\left.P S T_{\text {inicial }} / A F_{\text {inicial }}\right) x(\mathrm{a} / \mathrm{a}-1) / d d T_{1.2}$, donde $\mathrm{a}=\left(\ln P S T_{\text {final }}-\ln P S T_{\text {inicial }}\right) /\left(\ln A F_{\text {final }}-\ln \right.$ $A f_{\text {inicial }}$ ) y $d d T_{1.2}$ son los días entre el primer y el segundo muestreo indicados como inicial y final en la ecuación (Evans, 1972).

El diseño experimental empleado consistió en un arreglo factorial entre tres tipos de sustratos (TP; TPC y 
Tabla 1. Efecto de la adición del co-polímero sintético de propenamida-propeonato a diferentes sustratos sobre los parámetros de crecimiento de plantines de pimiento a los 57 días desde la siembra. Buenos Aires, Univ. Nac. de Lomas de Zamora, 1999.

\begin{tabular}{|c|c|c|c|c|c|c|c|c|}
\hline \multicolumn{2}{|c|}{ Tratamiento } & \multirow{2}{*}{$\begin{array}{l}\text { TRC } \\
\left(\text { dia }^{-1}\right)\end{array}$} & \multirow{2}{*}{$\begin{array}{l}\text { PFT } \\
\text { (mg) }\end{array}$} & \multirow{2}{*}{$\begin{array}{l}\text { PST } \\
(\mathrm{mg})\end{array}$} & \multirow{2}{*}{$\begin{array}{c}\mathrm{H} \\
(\mathrm{cm})\end{array}$} & \multirow{2}{*}{$\begin{array}{l}\text { DBT } \\
(\mathrm{mm})\end{array}$} & \multirow{2}{*}{$\begin{array}{l}\text { MS } \\
(\%)\end{array}$} & \multirow{2}{*}{ Rel V/R } \\
\hline Sustrato & Polímero & & & & & & & \\
\hline \multirow[t]{2}{*}{$\overline{\mathrm{TP}}$} & $\operatorname{Sin}$ & $0,0210 \mathrm{c}$ & $219,0 \mathrm{c}$ & $11,90 \mathrm{c}$ & $4,97 \mathrm{c}$ & $1,12 \mathrm{bc}$ & $5,26 \mathrm{~b}$ & $4,76 \mathrm{c}$ \\
\hline & Con & $0,0498 a$ & 774,4 a & $42,13 \mathrm{a}$ & $10,13 a$ & $2,25 \mathrm{a}$ & $6,00 \mathrm{~b}$ & $6,35 \mathrm{bc}$ \\
\hline \multirow[t]{2}{*}{ TPC } & Sin & $0,0414 \mathrm{~b}$ & $680,3 \mathrm{a}$ & $29,93 \mathrm{~b}$ & $7,53 \mathrm{~b}$ & $1,63 \mathrm{~b}$ & $4,00 \mathrm{c}$ & $8,16 \mathrm{~b}$ \\
\hline & Con & $0,0500 \mathrm{a}$ & 873,0 a & $45,23 a$ & $11,96 \mathrm{a}$ & $2,46 a$ & 7,65 a & $11,68 \mathrm{a}$ \\
\hline \multirow[t]{2}{*}{ Comercial } & Sin & $0,0361 \mathrm{~b}$ & $649,0 \mathrm{ab}$ & $31,62 a b$ & $9,73 a$ & $1,35 \mathrm{~b}$ & $8,61 \mathrm{a}$ & $8,68 a b$ \\
\hline & Con & 0,0468 a & $549,0 \mathrm{~b}$ & $36,50 \mathrm{a}$ & $9,45 a b$ & $1,98 \mathrm{a}$ & $9,01 \mathrm{a}$ & $9,31 \mathrm{a}$ \\
\hline
\end{tabular}

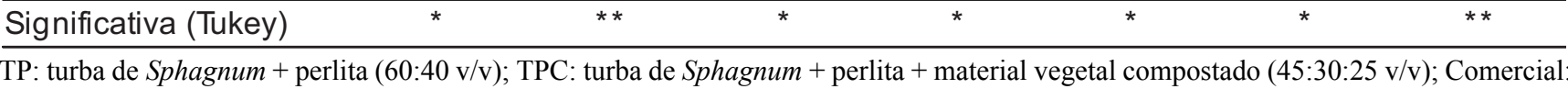
sustrato comercial en base a turba, perlita, compost (25\%) y vermiculita. TCR: Tasa de crecimiento relativa; PFT: Peso fresco total; PST: Peso seco total; H: Altura; DBT: Diámetro basal del tallo; MS: Contenido porcentual de materia seca; Rel V/R: Relación entre el peso seco del vástago y de la raíz.

Significancia de acuerdo a la prueba de Tukey: ${ }^{* *}$ : significativo al $1 \%, *$ : significativo al $5 \%$, ${ }^{\text {ns }}$ : no significativo.

Comercial) y la adición o no de polímeros superabsorbentes a cada uno de ellos con cuatro repeticiones, considerando a cada bandeja como una unidad experimental. Todos los datos obtenidos fueron sometidos a análisis de varianza con pruebas de Tukey al 5\% para las comparaciones entre medias de tratamientos y se utilizó regresión simple para establecer relaciones lineales entre variables de experimentación y de respuesta.

\section{RESULTADOS Y DISCUSIÓN}

El agregado del polímero produjo un aumento en la tasa de crecimiento de los plantines medida en base a peso seco entre los 9 y $57 \mathrm{dds}$, es decir, desde la emergencia hasta tamaño de transplante. El efecto sobre el crecimiento, sin embargo, fue diferente para los distintos sustratos empleados. Este aumento fue significativamente mayor en el caso de los plantines cultivados en el medio TP, sin materiales compostados en su composición. Si bien se registraron aumentos en el ritmo de crecimiento de los plantines cultivados en los medios TPC y Comercial, los mismos no resultaron tan importantes, por cuanto los tratamientos sin polímero para esos mismos sustratos mostraron tasas igualmente aceptables (Tabla 1).

El aumento en la tasa de crecimiento provocado por la adición del polímero a cualquiera de los tipos de sustratos

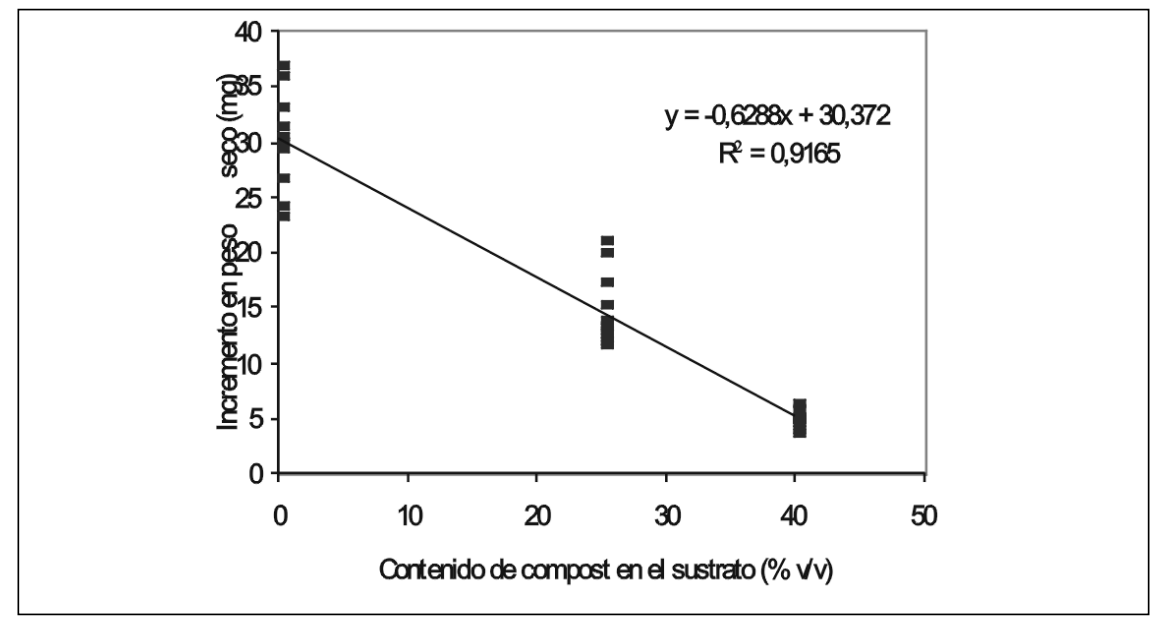

Figura 1. Relación entre el efecto de la adición del co-polímero de propenamida- propeonato al medio de crecimiento sobre el peso seco de plantines de pimiento a los 57 días desde la siembra, y el contenido porcentual de compost de los sustratos. Buenos Aires, Univ. Nac. de Lomas de Zamora, 1999.

empleados se manifestó además en la producción de plantines más pesados, más altos y con mayor diámetro basal de tallo (Tabla 1). Los pesos de los plantines, tanto en fresco como en seco, fueron significativamente mayores para el tratamiento con sustrato TPC, adicionado con polímero. El peso seco final de los plantines se incrementó afectado por el agregado del polímero en todos los sustratos evaluados. Sin embargo, este efecto fue menor a medida que aumentó el contenido de materiales compostados en el medio. La relación entre el efecto del agregado del polímero sobre el peso seco final de los plantines y el nivel de compost presente en el medio de cultivo resultó negativa $\left(r^{2}=0,92 ; p<0,05\right)$, de manera que el efecto del agregado del polímero sobre el peso seco final de los plantines disminuyó a razón de $0,6 \mathrm{mg}$ por unidad porcentual de compost contenido en el sustrato (Figura 1). La misma tendencia en los resultados se observó al analizar los componentes del crecimiento bajo condiciones térmicas contrastantes en etapas iniciales y un patrón similar para lo ocurrido con el peso en seco, corroborando lo observado en plantines de tomate por Johnson \& Piper (1997), quienes adicionaron diferentes tipos de polímeros a sustratos preparados con arena o compost como base de la mezcla. 
Tabla 2. Efecto de la adición del co-polímero sintético de propenamida-propeonato a diferentes sustratos sobre los parámetros de desarrollo foliar de plantines de pimiento a los 57 días desde la siembra. Buenos Aires, Univ. Nac. de Lomas de Zamora, 1999.

\begin{tabular}{|c|c|c|c|c|c|c|c|}
\hline \multicolumn{2}{|c|}{ Tratamiento } & \multirow{2}{*}{$\begin{array}{c}\mathrm{AF} \\
\left(\mathrm{cm}^{2}\right)\end{array}$} & \multirow{2}{*}{$\begin{array}{l}\text { DAF } \\
\left(\mathrm{cm}^{2}\right)\end{array}$} & \multirow{2}{*}{$\begin{array}{l}\text { TEFR } \\
\left(\mathrm{dia}^{-1}\right)\end{array}$} & \multirow{2}{*}{$\begin{array}{c}\text { AFE } \\
\left(\mathrm{cm}^{2} \mathbf{b}^{-1}\right)\end{array}$} & \multirow{2}{*}{$\begin{array}{c}\text { PAH } \\
\left(\mathrm{cm}^{2} \mathbf{g}^{-1}\right)\end{array}$} & \multirow{2}{*}{$\begin{array}{c}\text { TAN } \\
\left.\text { g. } \mathrm{cm}^{-2} \cdot \mathrm{dia}^{-1}\right) \\
\end{array}$} \\
\hline Sustrato & Polímero & & & & & & \\
\hline \multirow[t]{2}{*}{$\overline{\mathrm{TP}}$} & $\operatorname{Sin}$ & $28,89 \mathrm{c}$ & $268,0 \mathrm{c}$ & $0,103 \mathrm{~b}$ & $3175 \mathrm{bc}$ & 2938 b & $0,0104 \mathrm{c}$ \\
\hline & Con & 88,58 a & 743,5 a & $0,115 \mathrm{a}$ & $2803 \mathrm{c}$ & $2434 \mathrm{c}$ & $0,0232 a b$ \\
\hline \multirow[t]{2}{*}{ TPC } & $\operatorname{Sin}$ & $75,53 \mathrm{ab}$ & $661,9 a b$ & $0,110 a b$ & $3690 \mathrm{~b}$ & $2832 \mathrm{~b}$ & $0,0163 a b$ \\
\hline & Con & $91,81 \mathrm{~b}$ & $840,1 \mathrm{~b}$ & $0,105 b$ & $2866 \mathrm{c}$ & $2204 \mathrm{c}$ & 0,0296 a \\
\hline \multirow[t]{2}{*}{ Comercial } & $\operatorname{Sin}$ & $65,12 \mathrm{~b}$ & $601,3 \mathrm{~b}$ & $0,103 \mathrm{~b}$ & 5063 a & $3359 \mathrm{~b}$ & $0,0112 \mathrm{c}$ \\
\hline & Con & $61,81 \mathrm{~b}$ & $571,1 \mathrm{~b}$ & $0,104 \mathrm{~b}$ & 6021 a & 4148 a & $0,0077 \mathrm{c}$ \\
\hline \multicolumn{2}{|c|}{ Significativa (Tukey) } & ** & * & ** & * & * & * \\
\hline
\end{tabular}

TP: turba de Sphagnum + perlita (60:40 v/v); TPC: turba de Sphagnum + perlita + material vegetal compostado (45:30:25 v/v); Comercial: sustrato comercial en base a turba, perlita, compost (25\%) y vermiculita. AF: Area foliar; DAF: duración del área foliar; TEFR: Tasa de expansión foliar relativa; AFE: Area foliar específica (AF/Peso seco de hojas); PAH: Proporción areal de hojas (AF/Peso seco total); TAN: Tasa de asimilación neta.

Significancia de acuerdo a la prueba de Tukey: ${ }^{* *}$ : significativo al $1 \%, *$ : significativo al $5 \%$, ${ }^{\text {ns }}$ : no significativo.

Si bien el peso seco total sigue la tendencia del peso fresco total para los plantines cultivados en los medios TP y TPC, no ocurre lo mismo con los cultivados en sustrato comercial, los cuales no mostraron diferencias en peso fresco final por el agregado del polímero. Esto se vincula con el contenido hídrico de los plantines, que fue menor cuando se los cultivó en sustrato comercial y cuando se adicionaron polímeros a los medios TP y TPC (Tabla 1). Menores valores de MS (\%) estarían reflejando una mayor turgencia o una condición hídrica más favorable de los plantines y, de acuerdo con Reinink (1993), un mayor MS (\%) resulta indicativo de condiciones de crecimiento menos favorables.

La relación vástago/raíz fue afectada significativamente por la adición de polímeros a todos los sustratos, aunque el mayor efecto se observó en el caso de TPC (Tabla 1). Las relaciones más favorables para la supervivencia postransplante se observaron en los plantines cultivados en TP, con o sin el agregado del polímero. Este efecto aparece más asociado a aspectos físicos tales como el tipo de porosidad y la densidad aparente del medio de crecimiento radicular, vinculado a la resistencia que el mismo ofrece para su desarrollo (Thomas, 1993; Tittonell et al., 1999).

El comportamiento observado para la TCR se relaciona estrechamente $\left(r^{2}=\right.$
$0,83 ; \mathrm{p}<0,05)$ y en forma positiva con el desarrollo del área foliar experimentado por las plántulas, sugiriendo que un mayor crecimiento se encuentra más asociado a una mayor superficie fotosintetizante que a una mayor intensidad del proceso fotosintético. No obstante, éste último parámetro que fue caracterizado a través de la tasa de asimilación neta, resultó afectado favorablemente por la adición del polímero a los medios de crecimiento TP y TPC (Tabla 2), pero no así en el caso del sustrato comercial.

Los plantines cultivados sobre el medio de crecimiento TP mostraron una amplia diferencia en el desarrollo foliar por adición del polímero. Este efecto tuvo lugar en etapas más tardías en relación a los restantes tratamientos, lo cual se evidencia a través de una mayor tasa de expansión foliar relativa entre la primer y segunda fecha de muestreo. Las características del aparato fotosintético, sin embargo, mostraron diferente comportamiento entre tratamientos de sustratos. El área foliar de los plantines cultivados sobre sustrato comercial no fue favorablemente afectada por la adición de polímeros, pero éstos mostraron una mayor superficie fotosintetizante por unidad de biomasa total y de hojas (Tabla 2).

Se ha documentado que los polímeros hidrofílicos son capaces de mejorar la eficiencia en el uso del agua (Johnson \& Leah, 1990) aunque, en este ensayo, todos los tratamientos recibieron similares condiciones de irrigación, de manera que el patrón de uso de agua sólo puede reflejarse en los parámetros de tamaño, turgencia y peso seco del plantín. No obstante, además de mejorar la eficiencia hídrica, el polímero utilizado en este ensayo fue concebido para asegurar el aporte de nutrientes al medio. Esta característica parece confirmarse por cuanto su efecto sobre los parámetros de calidad del plantín fue menor cuando se lo adicionó a las mezclas conteniendo compost.

Resulta dificultoso distinguir el efecto de un mejor aprovechamiento del agua del de una mayor disponibilidad de nutrientes debido a que, según fue demostrado en diferentes trabajos (El Hady et al., 1981; Azzam, 1985; Widders, 1992), ambos factores se encuentran íntimamente relacionados. Probablemente, el principal efecto de los polímeros es disminuir la lixiviación de nutrientes desde la matriz del sustrato, debido a la mayor retención hídrica y a un aumento de la capacidad de intercambio de iones.

Así que, la adición del co-polímero de propenamida-propeonato a la mezcla empleada como sustrato para la producción de plantines de pimiento permitió mejorar la uniformidad y precocidad de los plantines, determinadas por una menor variabilidad en las características analizadas y un mayor tamaño de los 
mismos al momento del transplante, especialmente en las mezclas carentes de materiales que aportan capacidad de retención hídrica, tales como el compost.

Una menor relación vástago/raíz de los plantines de pimiento, es un factor de calidad indicativo de su tasa de recuperación postransplante. Esta relación no fue favorablemente afectada por la presencia del polímero en el medio de crecimiento, pero sí lo fue por el tipo de sustrato empleado. Las relaciones vástago/raíz más favorables se obtuvieron con el medio TP, con o sin polímero.

De acuerdo a los resultados del presente trabajo, el efecto de los polímeros sobre la tasa de crecimiento inicial de los plantines de pimiento es consecuencia de un mayor y más temprano desarrollo foliar, ya que la tasa de asimilación neta no resultó claramente afectada.

La adición del polímero utilizado resulta recomendable en un sustrato de bajo costo y fácil manipulación como el preparado sólo con turba y perlita, ya que los parámetros de calidad del plantín mejoran notablemente. Sin embargo, no es posible afirmar la conveniencia del empleo de estos aditivos cuando la mezcla a utilizar como sustrato posee propiedades hídricas y una dotación de nutrientes como las aportadas por la presencia de al menos $25 \%$ de materiales compostados.

\section{LITERATURA CITADA}

AZZAM, R.A. Tailoring polymeric gels for soil reclamation and hydroponics. Comm. Soil Sci. Plant Anal., v. 16, p. 1123-1138, 1985.

EL HADY, O.A.; TAYEL, M.Y.; LOTFY, A.A. Super gel as a soil conditioner. II: Its effects on plant growth, enzyme activity, water use efficiency and nutrient uptake. Acta Horticulturae, v. 119, p. 257-265, 1981

EL SAYED, H.; KIRKWOOD, R.C.; GRAHAM, N.B. The effects of a hydrogel polymer on the growth of certain horticultural crops under saline conditions. Journal of Experimental Botany, v. 42, p. 891-899, 1991.

EVANS, G.C. The quantitative analysis of plant growth. Blackwell Scientific Publications, Oxford 1972. 734 p.

HUNT, R. Plant growth analysis. Edward Arnold, London, 1978. $67 \mathrm{p}$

HUNT, R. Plant growth curves. The functional approach to plant growth analysis. Edward Arnold, London, 1982. 248 p.

JOHNSON, M.S. Effects of gel-forming polyacrylamides on moisture storage of sandy soils. J. Sc. of Food Agric., v. 35, p. 1196-1200, 1984.

JOHNSON, M.S.; LEAH, R.T. Effects of superabsorbent polyarylamides on efficiency of water use by crop seedlings. J. Sc. of Food Agric., v. 52, p. 431-434, 1990.

JOHNSON, M.S; PIPER, C.D. Cross-linked, water-storing polymers as aids to drought tolerance of tomatoes in growing media. J. Agronomy \& Crop Science, v. 178, p. 23-27, 1997.

KLASSEN, P. Transition to transplants. American Vegetable Grower, v. 41, p. 19-21, 1993.
LIPTAY, A.; NICHOLLS, S. Nitrogen supply during greenhouse transplant production affects subsequent tomato root growth in the field. Journal of the American Society for Horticultural Science, v. 118, p. 339-342, 1993.

ORZOLEK, M.D. Use of hydrophilic polymers in horticulture. Hort. Tech., v. 3, p. 421-444, 1993. PILL, W.G.; JACONO, C.G. Effects of hydrogel incorporation in peat-lite on tomato growth and water relations. Comm. Soil Sci. Plant Anal., v. 15, p. 799-810, 1984.

REININK, K. Relationship between effects of seasonal change on nitrate and dry matter content in lettuce. Scientia Horticulturae, v. 53, p. 35-44, 1993.

TAYEL, M.Y.; EL HADY, O.A. Super Gel as a soil conditioner. I: Its effects on water relations. Acta Horticulturae, v. 199, p. 247-256, 1981.

TAYLOR, K.C.; HALFACRE, R.G. The effect of hydrophlic polymer on media water retention and nutrient availability to Ligustrum lucidum. Horticultural Science, v. 21, p. 1159-1161, 1986. TITTONELL, P.A.; DE GRAZIA, J.; CHIESA, A. Calidad del plantín de pimiento (Capsicum annuиm L.) en función del volumen disponible para la exploración radiculary la porosidad del sustrato. Actas del XXII CONGRESO ARGENTINO DE HORTICULTURA, Septiembre de 1999, San Miguel de Tucumán, Argentina. I.S.B.N. N 987-97812-0-1, p. 31, 1999.

THOMAS, T.H. Effects of roots restriction and growth regulator treatments on the growth of carrot (Daucus carota L.) seedlings. Plant Growth Regulation, 13, 95-101, 1993.

VENUS, J.C.; CAUSTON, D.R. Plant growth analysis: a re-examination of the methods of calculation of relative growth and net assimilation rates without using fitted functions. Annals of Botany, v. 43, p. 633-638, 1979.

WIDDERS, I.E.; GARTON, R.W. Effects of preplant nutrient conditioning on elemental accumulation in tomato seedlings. Scientia Horticulturae, v. 52, p. 9-17, 1992. 